\title{
Use of Mobile Phone in Healthcare: Readiness among Urban Population of Puducherry, India
}

\author{
Mahendra M Reddy, Pruthu Thekkur, Marie Gilbert Majella, Kalaiselvi Selvaraj, \\ Jayalakshmy R, Sitanshu Sekhar Kar
}

\section{Mahendra M Reddy, Pruthu Thekkur, Marie Gilbert Majella, Kalaiselvi Selvaraj, Jayalakshmy R, Sitanshu Sekhar Kar}

Department of Preventive and Social Medicine, Jawaharlal Institute of Postgraduate Medical Education and Research

\section{Correspondence}

Dr. Jayalakshmy R, Department of Preventive and Social Medicine, $4^{\text {th }}$ floor, Admin block, JIPMER, Dhanavantri Nagar, Puducherry - 605006

Phone no: +91- 9944338145

Email: medico.jay@gmail.com

\section{History}

- Submission Date: 06-09-15;

- Review completed: 31-01-16:

- Accepted Date: 07-02-16.

DOI : 10.5530/ijmedph.2016.2.8

Article Available online

http://www.ijmedph.org/v6/i2

\section{Copyright}

(c) 2016 Phcog.Net. This is an open-access article distributed under the terms of the Creative Commons Attribution 4.0 International license.

\begin{abstract}
Introduction: Use of mobile phones in planning of health care interventions is on the rise. Before planning of such interventions, it is essential to know about readiness among the target population.

Objective(s): To identify the access to personal mobile phone, knowledge on usage in terms of making/receiving call/SMS and the willingness to use mobile phone as a medium for health communication among adults in an urban area of Puducherry, South India.

Methodology: A community based cross-sectional study was conducted in two randomly selected areas out of the four in the urban field practice area of a medical college in Puducherry during July, 2014. Systematic random sampling was done to select 126 houses. All individuals above 18 years in those households were included. A pre-tested interview schedule was used to collect information. Association between socio-demographic factors and willingness to receive health tip through mobile phone was found using binary logistic regression.

Results: Among 299 participants interviewed, 227 (75.9\%) had personal mobile phones; $229(76.6 \%), 128(42.8 \%)$ and $131(43.8 \%)$, were able to receive and make a call, read SMS, and text SMS respectively. Nearly $50 \%$ were willing to receive health information through mobile phones. Of these, $67.5 \%$ were willing to receive through call/SMS and $35.5 \%$ only through calls. On multivariate binary logistic analysis, individuals having personal mobile and younger age were found to be willing to receive health information through mobile phones.

Conclusion: Three in every five individuals having personal mobile were willing to receive health information through mobile with most of them preferring call/SMS over only call.

Key words: Health information, mHealth, Mobile phone, Readiness.
\end{abstract}

\section{INTRODUCTION}

Mobile health (mHealth) broadly encompasses the use of mobile telecommunication and multimedia technologies in health care delivery systems. ${ }^{1}$ Mobile phones are now widely used to reduce the barriers and also to bridge the gaps in healthcare. mHealth use is focussed in many areas such as treatment compliance, health promotion, health service reporting and monitoring and also in training of health workers. ${ }^{1}$ Though mHealth has been tested and adapted in healthcare delivery in developed countries, its utility in developing countries is still questionable. A developing country like India has over 970 million wireless subscribers, which is more than $75 \%$ of its total population. ${ }^{2}$ This justifies the rise in interventions planned using mobile phones in healthcare system. With about $58 \%$ of the wireless subscribers residing in urban areas, the interventions using mHealth are tried more in urban areas. ${ }^{2}$ But, studies regarding the knowledge of the mobile phone users and their preferred mode and willingness to use mHealth are scarce. Before planning for any newer interventions using technology like mobile phones as the channel of communication, receiver (general population) level readiness needs to be assessed to know its effectiveness in practice. ${ }^{3}$

With this intention, this study was planned among adults residing in an urban area of Puducherry, South India, to identify the access to personal mobile phone, knowledge on usage in terms of making/ receiving call/SMS and their willingness to use mobile as a medium for health communication. This study also tried to find out the extent of mHealth reach in this community.

\section{METHODS}

A community based cross-sectional study was conducted among adults more than 18 years of age in the urban field practice area of a medical college, Puducherry. This study was done during the month of July, 2014. The Urban Health Centre provides
Cite this article : Reddy MM, Thekkur P, Majella MG, Selvaraj K, Jayalakshmy R, Kar SS. Use of Mobile Phone in Healthcare: Readiness among Urban Population of Puducherry, India. Int. J. Med. Public Health, 2016; 6(2):94-97. 
comprehensive health care services to about 9500 population spread over four urban wards.

As no related previous published literature was available, sample size was calculated assuming that $50 \%$ of the population will be willing to receive health information through mobile phones to give the largest sample size. The study unit was an individual but it was decided to consider the households as the sampling unit and include all adults in the selected households. Therefore with $8 \%$ absolute precision, 95\% CI and design effect of 2 , the minimum sample size was calculated using nMaster 2.0 to be 312 individuals. Considering the average family size of 4.5 in Puducherry, it was expected to find 5 adults in 2 households, giving a sample size of 125 households for the study.

Two out of the four areas (with a total of around 632 households in the two selected areas) were selected randomly. Eligible households were covered by systematic random sampling. Starting from the random number of four, subsequent every $5^{\text {th }}$ house was approached for the study. All adults more than 18 years residing in the house at the time of interview were included in the study. In case an adult was not present in the house at time of interview, subsequent visits to same house was done to make sure all those eligible in each selected house were included in the study.

After obtaining verbal consent, interview was held using a semistructured, pre tested interview schedule. The interview schedule consisted of questions regarding the socio-demographic status, presence of mobile phone, knowledge regarding the use of mobile phone in terms of making/receiving call/SMS, the willingness to receive health information and their preferred mode to receive health information. Participants having personal mobile phones were asked whether they had received any form of health tips through their mobile phones in the period one month before the study.

The data were entered in Microsoft Excel and analyzed using SPSS version 20. The continuous data like age was converted into categorical data according to relevance. All the categorical data were presented as percentages. Association of willingness to receive health information using mobile and socio-demographic factors were tested using chi-square test followed by multivariate analysis using binary logistic regression. The associations were summarised in terms of odds ratio with $95 \%$ CI.

\section{RESULTS}

A total of 299 adults were interviewed among the 126 eligible households. Out of 299 participants, 167 (55.8\%) were females and 162 (54.1\%) were aged between 30 to 60 years. Around half of the study participants, 149 (49.8\%) had completed at least eight years of schooling and 146 (48.8\%) were currently employed.

Of the total 299 study participants, 227 (75.9\%) had access to personal mobile phone. Among the 299 interviewed, 229 (76.6\%), 128 (42.8\%), 131 (43.8\%), were able to receive and make a call, read SMS, and text SMS respectively. Figure 1 shows the proportion of people having access to personal mobile and their willingness to receive health information through mobile. A total of 148 (49.5\%) wanted health information through mobile. Among the 227 who had personal mobile phone, 142 $(62.6 \%)$ were willing to receive health information through mobile and of these only $15(10.6 \%)$ had received some form of health tips through mobile phones in the last one month prior to the study. Of the 148 who were willing to receive health information, the preferred modes for health communications were call/SMS in 97 (65.5\%) and only call in $51(34.5 \%)$. Majority were willing to receive information regarding non-communicable diseases 94 (63.5\%) and healthy lifestyle 61 (41.2\%). Table 1 shows association of socio-demographic factors with willingness to receive health information through mobile phone. On multivariate

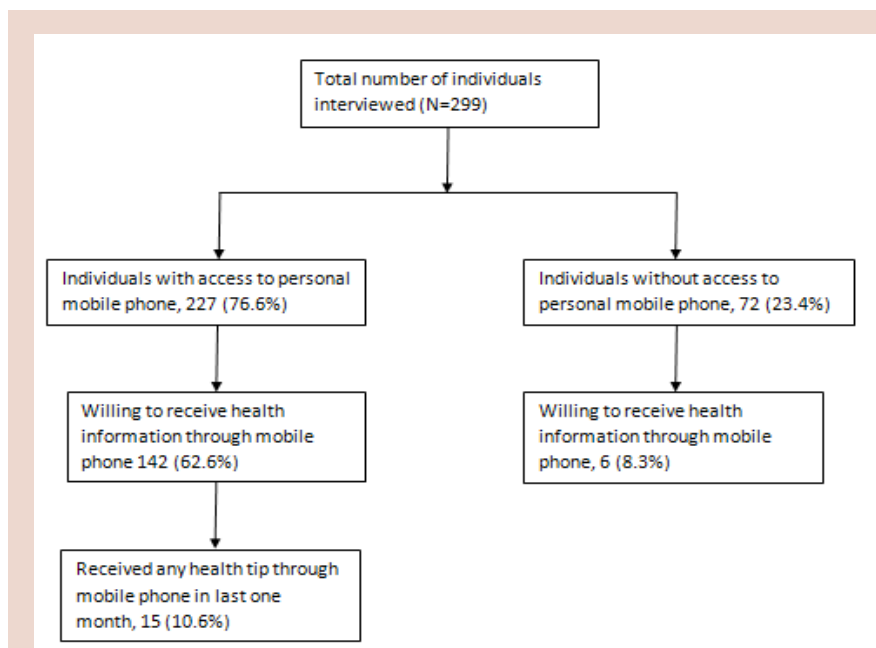

Figure 1: Access to personal mobile phone and willingness to receive health tip through mobile phone among adults of selected urban population of Puducherry $(\mathrm{N}=299)$

analysis using binary logistic regression, individuals with access to personal mobile phone were found to be willing to receive health information compare to those who did not have personal mobile phone (OR with 95\% CI: 12.2 (4.8-31.1)). Similarly, younger age was found to be independently associated with willingness to receive health information through mobile phone compare to those aged 60 years and above, OR with 95\% CI: for age group 18-29 years it was $5.6(2.1-14.9)$ and for age group 30-44 years it was 2.7 (1.1-6.6).

\section{DISCUSSION}

In the current study conducted in a selected urban locality of Puducherry, more than two-thirds of the study participants had access to personal mobiles. A study from Bangalore among general population showed that $87 \%$ had access to personal mobiles. ${ }^{4}$ Another study in Tamil Nadu, India done among tuberculosis patients reported that $68 \%$ had access to mobile phones. ${ }^{5}$ Studies from other countries also had focussed on a specific disease groups, have reported personal mobile phone ownership ranging from $61.2 \%$ to $74 \% .^{6-9}$ Though, high penetration of personal mobile phones was seen in the current study (75.9\%), it was comparatively lower than Bangalore study. The difference noted may be due to the fact that, Bangalore being a hub of technological boom in India, is expected to have higher access to personal mobile phone.

In our study, about three in every five individuals having a personal mobile were willing to receive health information through mobile with most of them preferring either call or SMS over only call. The willingness to receive health information through mobile phones was around 99\% in the study done in Bangalore which was much higher compared to our study. ${ }^{4}$ Similarly a study done among those attending public run sexual health youth clinics in Canada showed $73 \%$ willingness in using mHealth services. ${ }^{10}$ The difference may be due to difference in study settings and also the proficiency of knowledge on usage which drives the confidence to use mHealth. These differences also point the need in conducting the regional specific studies to know the readiness levels before planning for mHealth based interventions.

In the Bangalore study $89 \%$ preferred only call and in another study from Karachi, Pakistan among hypertensive patients, $64.6 \%$ preferred only call 
Table 1: Association of Socio demographic factors with willingness to receive health information through mobile phone among adults of selected urban population of Puducherry $(\mathrm{N}=299)$

\begin{tabular}{|c|c|c|c|c|}
\hline Socio-demographic details & Total, $\mathrm{N}$ & Willing for mHealth, $n(\%)$ & Bivariate odds ratio & Adjusted odds ratio \\
\hline & 299 & $148(49.5)$ & $(95 \% \mathrm{CI})$ & $(95 \% \mathrm{CI})$ \\
\hline \multicolumn{5}{|l|}{ Age (in years) } \\
\hline $18-29$ & 79 & $55(69.6)$ & $11(4.8-25.3)$ & $5.6(2.1-14.9)$ \\
\hline $45-59$ & 65 & $25(38.5)$ & $3(1.3-7.0)$ & $1.5(0.6-3.9)$ \\
\hline$\geq 60$ & 58 & $10(17.2)$ & 1 & 1 \\
\hline Female & 167 & $72(43.1)$ & 1 & 1 \\
\hline \multicolumn{5}{|c|}{ Educational status (Years of schooling) } \\
\hline$\leq 8$ & 150 & $56(37.3)$ & 1 & 1 \\
\hline$>8$ & 149 & $92(61.7)$ & $2.7(1.7-4.3)$ & $0.8(0.5-1.5)$ \\
\hline \multicolumn{5}{|l|}{ Occupational status } \\
\hline No & 72 & $6(8.3)$ & 1 & 1 \\
\hline
\end{tabular}

as a preferred option to receive health information. ${ }^{4,9}$ In our study the proportion of people preferring only call was comparatively less and this may be due to the difference in the study population. The participants in the current study had, higher proportion of young participants, better education status and almost $40 \%$ were able to read and text message. The study among hypertensive patients, with majority aged above 45 years had showed higher preference for voice calls.

In our study majority were willing to receive information regarding non-communicable diseases (63.5\%) and healthy lifestyle (41.2\%). The study done in Bangalore showed preference to information on healthy lifestyles (35\%), maternal and child health (MCH) $(27 \%)$ and nutrition (25\%). ${ }^{4}$ The preponderance to receive information on $\mathrm{MCH}$ and nutrition could be due to two-thirds of study participants being females in the Bangalore study.

The study has few strengths. It is the first study in general population from an urban area in India which tried to look at the readiness for mHealth usage. With this study we have identified the readiness of general public to receive health information through mobile. This study also has captured the reach of mHealth in the community under study.

The study has few limitations. The study does not focus on any particular group or target audience for special interventions such as in chronic disease patients, adolescents, pregnant mothers which can be done in future. In our study we have not tried to see the preferred language for SMS which could have further helped in planning of health message delivery. The study results cannot be generalizable to other urban areas in the state or country as the readiness levels change in accordance with the accessibility and usage of mobile phones.

The study has few implications. From this study it has been shown people living in an urban area in India under study are willing to use either call or SMS compare to only call as in the rural area as shown in study from Bangalore. As the cost of SMS is comparatively lesser than that of call, this method can now be tried as part of health promotion campaigns. In our study although the penetrance of mobile phone is high, the readiness in use of mHealth is on the lower side. The study calls for efforts to increase awareness of mHealth in community before planning for any intervention based on it. Qualitative studies have to be done in order to find the reasons for low willingness for mHealth among the study population. Our study shows that people in younger age group and having personal mobile are willing to receive health information through mobile. Thus, health information regarding healthy lifestyle practices and preventing NCDs can be sent to these people with younger age.

\section{CONCLUSION}

In our study more than two-thirds had access to personal mobiles and knew how to receive and make a call whereas about two-fifth knew how to read/text SMS. Three in every five individuals having personal mobile were willing to receive health information through mobile with most of them preferring call/SMS over only call.

\section{ACKNOWLEDGEMENT}

We acknowledge the help of MBBS trainees- Vivot, Koninica, Krishna Sai, Yeshashvi, Kilambi, Jyoti Puja, KojYasa and JyotiBaghela for their help in data collection and data entry. Also, authors acknowledge help of medical officer of UHC, Dr. Jagadeeshwaran for allowing to carry out this study.

\section{REFERENCES}

1. World Health Organization. Towards the development of an mHealth strategy: A literature review. WHO 2007. Available from: http://www.who.int/goe/mobile health/mHealthReview_Aug09.pdf. [Last accessed on 2015 Sep 6]

2. Highlights on telecom subscription data as on 31st March, 2015. Telecom Regulatory Authority of India 2015. Available from: http://www.trai.gov.in/ WriteReadData/PressRealease/Document/PR-34-TSD-Mar-12052015.pdf. [Last accessed on 2015 Sep 6]

3. World Health Organisation. mHealth: New horizons for health through mobile technologies: second global survey on eHealth. Global Observatory for eHealth series Volume-3. WHO 2011. Available from: http://www.who.int/goe/ publications/goe_mhealth_web.pdf. [Last accessed on 2015 Sep 6]

4. DeSouza SI, Rashmi MR, Vasanthi AP, Joseph SM, Rodrigues R. Mobile Phones: The Next Step towards Healthcare Delivery in Rural India? Lovis C, editor. PLoS ONE 2014;9(8):e104895.

5. Elangovan R, Arulchelvan S. A Study on the Role of Mobile Phone Communication in Tuberculosis DOTS Treatment. Indian J Community Med Off Publ Indian Assoc Prev Soc Med 2013;38(4):229-33.

6. Okoro E, Sholagberu H, Kolo P. Mobile phone ownership among Nigerians with diabetes. Afr Health Sci 2010;10(2):183-6. 
7. Afshar AE, Weaver RG, Lin M, Allan M, Ronksley PE, Sanmartin C, et al. Capacity and willingness of patients with chronic noncommunicable diseases to use information technology to help manage their condition: a cross-sectional study. CMAJ Open 2014;2(2):E51-59

8. Zurovac D, Otieno G, Kigen S, Mbithi AM, Muturi A, Snow RW, et al. Ownership and use of mobile phones among health workers, caregivers of sick children and adult patients in Kenya: cross-sectional national survey. Glob Health 2013;9:20.
9. Hussain IM, Naqvi BS, Ahmed I, Ali N. Hypertensive patients' readiness to use of mobile phones and other information technological modes for improvingtheir compliance to doctors' advice in Karachi. Pak J Med Sci 2014;31(1).

10. Symonds L. Youth's acceptance of mobile phone text messaging for STI health promotion [Dissertation] The University of British Columbia 2013. Available from: https://circle.ubc.ca/handle/2429/44181. [Last accessed on 2015 Sep 6]

Cite this article : Reddy MM, Thekkur P, Majella MG, Selvaraj K, Jayalakshmy R, Kar SS. Use of Mobile Phone in Healthcare: Readiness among Urban Population of Puducherry, India. Int. J. Med. Public Health, 2016; 6(2):94-97. 